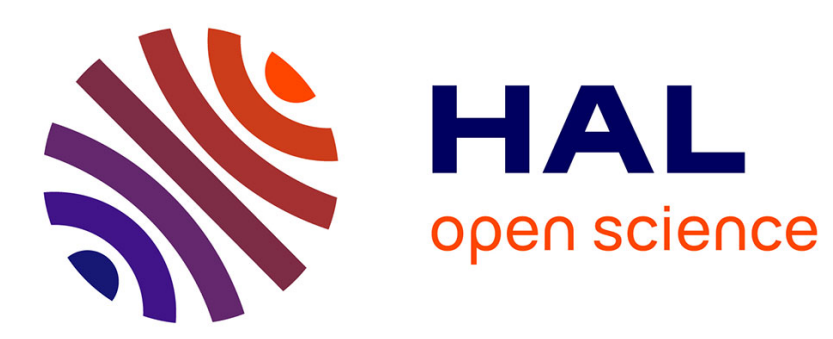

\title{
Formation et confinement d'ions multicharges dans un champ quadrupolaire a haute fréquence
}

\author{
C. Schwebel, P.A. Môller, Pham Tu Manh
}

\section{To cite this version:}

C. Schwebel, P.A. Môller, Pham Tu Manh. Formation et confinement d'ions multicharges dans un champ quadrupolaire a haute fréquence. Revue de Physique Appliquée, 1975, 10 (4), pp.227-239. 10.1051/rphysap:01975001004022700 . jpa-00243908

\section{HAL Id: jpa-00243908 https://hal.science/jpa-00243908}

Submitted on 1 Jan 1975

HAL is a multi-disciplinary open access archive for the deposit and dissemination of scientific research documents, whether they are published or not. The documents may come from teaching and research institutions in France or abroad, or from public or private research centers.
L'archive ouverte pluridisciplinaire HAL, est destinée au dépôt et à la diffusion de documents scientifiques de niveau recherche, publiés ou non, émanant des établissements d'enseignement et de recherche français ou étrangers, des laboratoires publics ou privés. 


\title{
FORMATION ET CONFINEMENT D'IONS MULTICHARGES DANS UN CHAMP QUADRUPOLAIRE A HAUTE FRÉQUENCE
}

\author{
C. SCHWEBEL, P. A. MÖLLER et PHAM TU MANH \\ Institut d'Electronique Fondamentale, Bât. 220, Université Paris-XI, \\ 91405 Orsay, France
}

(Reçu le 3 janvier 1975, accepté le 14 mars 1975)

\begin{abstract}
Résumé. - On montre la possibilité de créer et de confiner des ions d'état de charge élevé avec une cage quadrupolaire en haute fréquence. Il est d'abord fait un bref rappel du principe de fonctionnement, puis sont ensuite dégagées les conditions optimales pour la formation et le piégeage des ions multichargés. La seconde partie de l'article décrit l'appareillage expérimental et expose les résultats obtenus ; ainsi, pour la première fois, des ions multichargés ont pu être confinés et détectés dans un tel système.
\end{abstract}

Abstract. - It is shown that production and storage of multiply charged ions are possible with a three-dimensional RF quadrupole ion trap. The theoretical basis for the trapping of charged particles is first briefly examined and the dependence of ionization on the phase of the RF field is examined in order to derive the optimum operating modes for the creation of highly charged ions. The second part of the paper is devoted to the description of the experimental apparatus, and results are reported, showing for the first time the storage and detection of multiply charged ions with such a system.

1. Introduction. - Les travaux de W. Paul et coll. $[1,2]$ ont montré que les particules chargées peuvent être piégées et confinées par un champ électrique quadrupolaire haute fréquence. Une caractéristique de ce confinement est qu'il peut être rendu sélectif, les particules ayant un rapport $(Z e / M)$ de valeur convenable étant seules confinées, d'où l'emploi actuel de pièges quadrupolaires comme filtres de masse $[2,3,4]$. Nous avons envisagé une autre application de ce dispositif en vue de la formation d'ions d'état de charge élevé $(Z \gg 1)$, par bombardement électronique. Pour cela, il faut que des ions de charge faible soient maintenus dans un volume donné pendant un temps suffisamment long pour que l'ionisation pas à pas puisse se produire, grâce à des chocs électroniques successifs. A notre connaissance, une telle étude n'a encore jamais été faite, tous les travaux réalisés à ce jour sur le confinement d'ions par champ quadrupolaire haute fréquence ne concernant que des ions monochargés.

Nous commencerons par rappeler brièvement le principe de ce type de confinement, en nous efforçant de dégager les conditions optimales pour la création et le piégeage des ions multichargés. Nous exposerons ensuite le travail expérimental et les résultats obtenus.
2. Aspects théoriques. -2.1 DÉfINITION DU PROBLÈME. EQUATIONS DU MOUVEMENT. - Les forces agissant sur les particules chargées doivent être sinusoïdales dans le temps et leur amplitude en un point proportionnelle à la distance à un centre-origine. Dans le cas le plus général d'une configuration tridimensionnelle, le potentiel en chaque point, s'écrit :

$$
\Phi=\varphi(t) \cdot\left(\alpha_{x} x^{2}+\alpha_{y} y^{2}+\alpha_{z} z^{2}\right) .
$$

S'il n'y a pas de charge libre à l'intérieur, le potentiel vérifie l'équation de Laplace ; on en déduit :

$$
\alpha_{x}+\alpha_{y}+\alpha_{z}=0 .
$$

Par ailleurs, si le système admet un axe $O z$ de symétrie de révolution, on a les relations :

$$
\begin{aligned}
& \alpha_{x}=\alpha_{y}=\alpha \\
& \alpha_{z}=-2 \alpha
\end{aligned}
$$

d'où :

$$
\Phi=\Phi_{0}\left(x^{2}+y^{2}-2 z^{2}\right)=\Phi_{0}\left(r^{2}-2 z^{2}\right) .
$$

La forme de $\Phi(r, z)$ donné par (1) montre que les surfaces équipotentielles sont des hyperboloïdes de révolution autour de $O z$, dont certains peuvent être 
matérialisés par des surfaces conductrices. Le système le plus simple permettant d'obtenir la répartition de potentiel convenable se compose de trois électrodes. Dans un plan de section méridien $(r, z)$, la section de ces électrodes correspond à des hyperboles complémentaires, et les conditions aux limites donnent (Fig. 1)

$$
r_{0}^{2}=2 z_{0}^{2}
$$

où $r_{0}$ désigne le rayon de l'électrode centrale et $2 z_{0}$ la distance minimale séparant les deux électrodes extrêmes ou chapeaux.

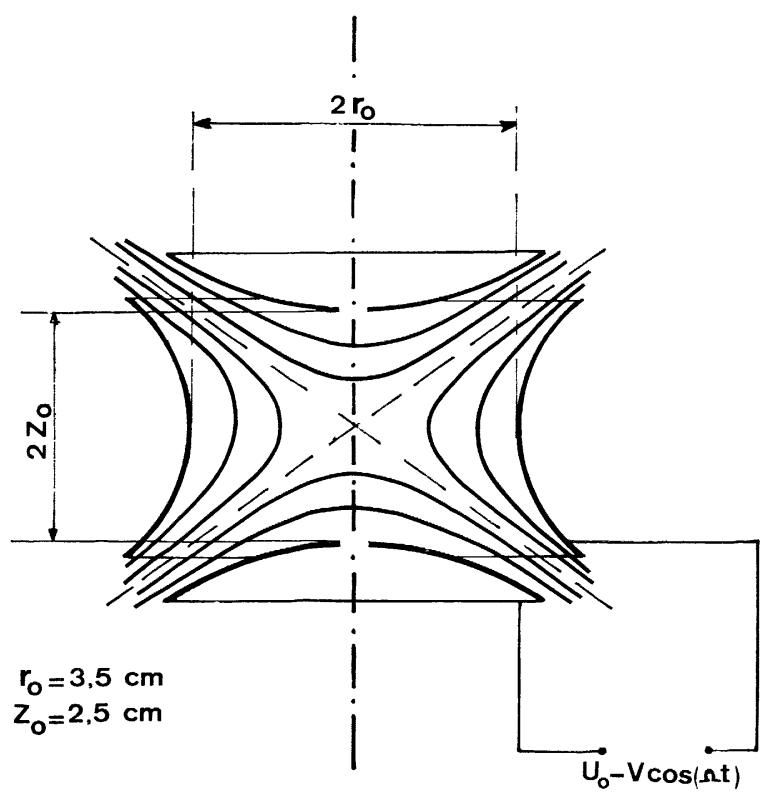

FIG. 1. - Représentation de la cage quadrupolaire et des lignes équipotentielles.

Soit

$$
\Phi_{0}=U_{0}-V \cos \Omega t
$$

le potentiel appliqué entre l'électrode centrale et les chapeaux. $U_{0}$ et $V \cos \Omega t$ sont respectivement des tensions continue et alternative. Le potentiel en chaque point, mesuré par rapport au potentiel existant au centre $O$ du système, s'écrit :

$$
\Phi=\left(U_{0}-V \cos \Omega t\right) \frac{r^{2}-2 z^{2}}{4 z_{0}^{2}} .
$$

On en dérive les expressions des composantes du champ électrique :

$$
\begin{aligned}
& E_{r}=-\left(U_{0}-V \cos \Omega t\right) \frac{r}{2 z_{0}^{2}} \\
& E_{z}=\left(U_{0}-V \cos \Omega t\right) \frac{z}{z_{0}^{2}} .
\end{aligned}
$$

Les équations (2) montrent que :

- quand le champ focalise en $r$, il défocalise en $z$, et inversement ;

- la force de rappel est deux fois plus forte en $z$ qu'en $r$;
Les équations du mouvement pour une particule de charge $Z e$ et de masse $M$, s'écrivent :

$$
\begin{aligned}
& \frac{\mathrm{d}^{2} r}{\mathrm{~d} t^{2}}+\frac{Z e}{2 M z_{0}^{2}}\left(U_{0}-V \cos \Omega t\right) r=0 \\
& \frac{\mathrm{d}^{2} z}{\mathrm{~d} t^{2}}-\frac{Z e}{M z_{0}^{2}}\left(U_{0}-V \cos \Omega t\right) z=0 .
\end{aligned}
$$

Ces deux équations sont découplées. Elles peuvent se mettre sous la forme d'équations de Mathieu :

$$
\frac{\mathrm{d}^{2} u}{\mathrm{~d} \xi^{2}}+(a-2 q \cos 2 \xi) u=0
$$

si l'on pose :

$$
\begin{aligned}
& u=r \text { ou } z \\
& \xi=\frac{\Omega t}{2}
\end{aligned}
$$

avec

$$
\begin{aligned}
& a_{z}=-2 a_{r}=-\frac{4 Z e U_{0}}{M z_{0}^{2} \Omega^{2}} \\
& q_{z}=-2 q_{r}=-\frac{2 Z e V}{M z_{0}^{2} \Omega^{2}} .
\end{aligned}
$$

2.2 Diagramme De STABilité. - La solution générale est de la forme :

$u=A \mathrm{e}^{\mu \xi} \sum_{n=-\infty}^{\infty} C_{2 n} \mathrm{e}^{j 2 n \xi}+B \mathrm{e}^{-\mu \xi} \sum_{n=-\infty}^{\infty} C_{2 n} \mathrm{e}^{-j 2 n \xi}$.

Les quantités $\mu$ et $C_{2 n}$ sont fonctions des paramètres $a$ et $q$ de (4). Le paramètre $\mu$ est important car il détermine la stabilité de la particule dans le champ :

- si $\mu$ est réel ou complexe : l'amplitude de $u$ croît indéfiniment et la trajectoire est dite instable;

- si $\mu$ est imaginaire, $\mu=j \beta$ avec $\beta$ réel $\neq$ nombre entier : l'amplitude du mouvement est bornée ; il y a alors stabilité.

En traçant dans le plan $(a, q)$ les courbes $\mu(a, q)=$ Cte, on obtient des régions du plan correspondant à $\mu$ imaginaire pur ou complexe, c'est-à-dire à des solutions stables ou instables. Par superposition des deux diagrammes obtenus pour $r$ et $z$, nous obtenons des portions du plan $(a, q)$, donnant la stabilité simultanément en $r$ et en $z$ (Fig. 2). A noter que les coordonnées des deux diagrammes, qui admettent la droite $q=0$ comme axe de symétrie, sont dans un rapport $(-2)$. On peut distinguer plusieurs zones de stabilité (notées I, II, III,... sur la Fig. 2), mais seule la zone I est intéressante car d'aire assez importante pour recevoir une application pratique. Cette zone, dont la figure 3 donne une reproduction agrandie, est délimitée par les lignes $\beta_{z}=0$, $\beta_{z}=1, \beta_{r}=0, \beta_{r}=1$. En la coupant par une droite $D$ de pente fixe $a / q$, on définit un domaine de fréquence $\Omega$ où une particule de rapport $\eta=Z e / M$ donné est confi- 


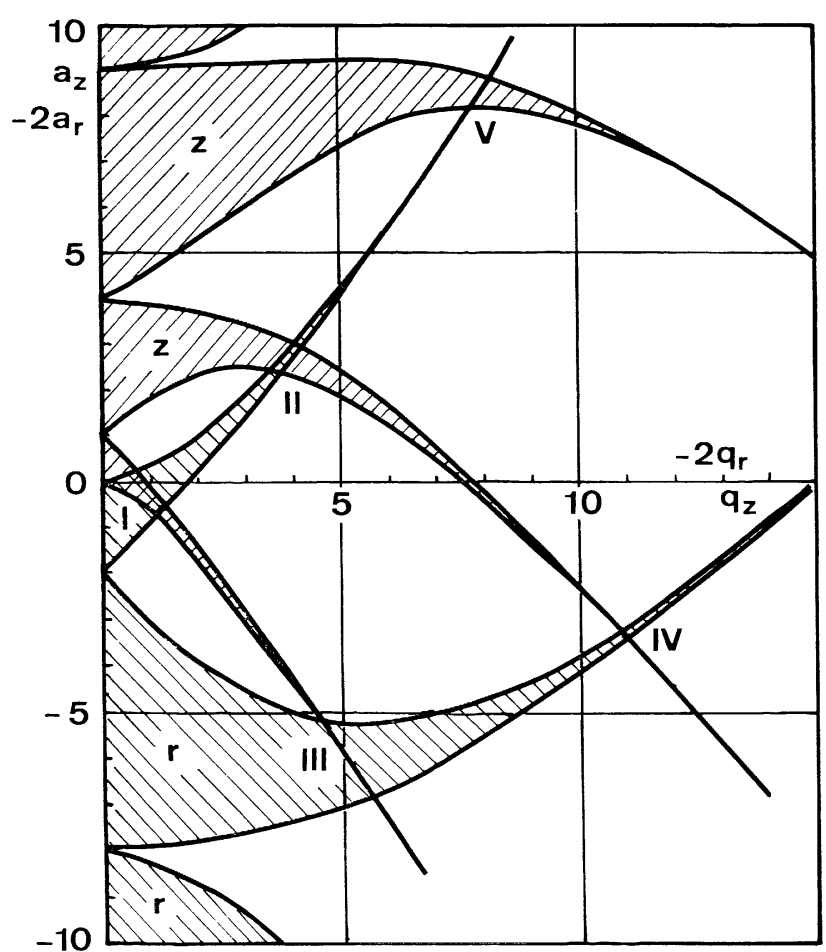

FIG. 2. - Diagramme de stabilité en $r$ et $z$, montrant plusieurs zones stables.

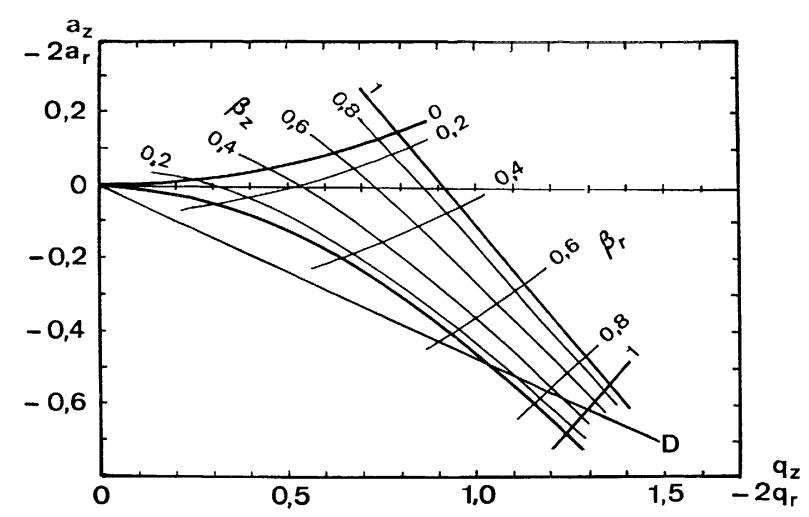

FIG. 3. - Diagramme principal de stabilité, correspond à la zone I de la figure 2.

née, c'est-à-dire dont l'amplitude du mouvement reste limitée. En particulier, si la pente $a / q=2 U_{0} / V$ est choisie telle que $D$ coupe la zone stable près du point extrême $\left(\beta_{z}=0, \beta_{r}=1\right)$, le domaine $\eta$ sera très réduit et le système fonctionnera en spectromètre de masse de haute résolution.

La droite $a=0\left(U_{0}=0\right)$ correspond au plus grand domaine possible en $\eta$. A la valeur maximum de $q$ :

$$
q_{\max }=0,908
$$

correspond une valeur minimum pour $\Omega$, d'après (4) :

$$
\Omega_{\min }=1,484\left(\frac{Z e}{M} \cdot \frac{2 V}{z_{0}^{2}}\right)^{1 / 2} .
$$

REVUE de PHYSIQUe APPLIQUÚe. - T. 10, No 4, JUILLET 1975
2.3 Solution apProchéE. Matrice DE transfert. — Dans la zone de stabilité où $\mu=j \beta, \beta$ réel, on peut réécrire (5) sous la forme :

$$
u=\alpha_{1} u_{1}(t)+\alpha_{2} u_{2}(t)
$$

avec

$$
\begin{aligned}
& u_{1}(t)=\sum_{-\infty}^{\infty} C_{2 n} \cos (2 n+\beta) \xi \\
& u_{2}(t)=\sum_{-\infty}^{\infty} C_{2 n} \sin (2 n+\beta) \xi
\end{aligned}
$$

$\alpha_{1}$ et $\alpha_{2}$ sont des constantes définies par les conditions initiales existant à l'instant $t_{0}$ de la création de l'ion, c'est-à-dire par $u_{0}, \dot{u}_{0}$ et la phase $\varphi_{0}$ du champ $\mathrm{HF}\left(\varphi_{0}=\Omega t_{0}\right)$ :

$$
\begin{aligned}
& u_{0}\left(\varphi_{0}\right)=\alpha_{1} u_{1}\left(\varphi_{0}\right)+\alpha_{2} u_{2}\left(\varphi_{0}\right) \\
& \dot{u}_{0}\left(\varphi_{0}\right)=\alpha_{1} \dot{u}_{1}\left(\varphi_{0}\right)+\alpha_{2} \dot{u}_{2}\left(\varphi_{0}\right) .
\end{aligned}
$$

Les coefficients $\alpha_{1}$ et $\alpha_{2}$ se déduisent de (8) :

$$
\begin{aligned}
& \alpha_{1}=\frac{1}{W}\left|\begin{array}{ll}
u_{0} & u_{2}\left(\varphi_{0}\right) \\
\dot{u}_{0} & \dot{u}_{2}\left(\varphi_{0}\right)
\end{array}\right| \\
& \alpha_{2}=\frac{1}{W}\left|\begin{array}{ll}
u_{1}\left(\varphi_{0}\right) & u_{0} \\
\dot{u}_{1}\left(\varphi_{0}\right) & \dot{u}_{0}
\end{array}\right|
\end{aligned}
$$

avec

$$
W=u_{1}\left(\varphi_{0}\right) \dot{u}_{2}\left(\varphi_{0}\right)-u_{2}\left(\varphi_{0}\right) \dot{u}_{1}\left(\varphi_{0}\right) .
$$

Les équations du mouvement ne possédant pas de termes d'amortissement, $W$ est une constante du mouvement.

D'après (7), les fréquences d'oscillation ionique sont données par :

$$
\omega_{n}=(2 n+\beta) \frac{\Omega}{2}
$$

et la fréquence fondamentale est :

$$
\omega_{r_{0}}=\frac{1}{2} \Omega \beta_{r}, \quad \omega_{z_{0}}=\frac{1}{2} \Omega \beta_{z} .
$$

En explicitant les relations (6), on peut écrire : $u=\left(\alpha_{1} \cos \omega_{0} t+\alpha_{2} \sin \omega_{0} t\right) \times$

$$
\begin{aligned}
& \times\left[C_{0}+\sum_{1}^{\infty}\left(C_{2 n}+C_{-2 n}\right) \cos (n \Omega t)\right] \\
& -\left(\alpha_{1} \sin \omega_{0} t-\alpha_{2} \cos \omega_{0} t\right) \\
& \times\left[\sum_{1}^{\infty}\left(C_{2 n}-C_{-2 n}\right) \sin (n \Omega t)\right] .
\end{aligned}
$$

Les constantes $C_{2 n}$ et $C_{-2 n}$ ne dépendent que de $a$ et $q$ et on peut les calculer à partir de relations de récurrence [8]. On trouve alors que les termes

$$
\sum_{n}\left(C_{2 n}+C_{-2 n}\right) \text { et } \sum_{n}\left(C_{2 n}-C_{-2 n}\right)
$$

sont très petits devant $C_{0}$ pour $q \leqslant 0,4$, c'est-à-dire que le premier terme de (11) est prépondérant. D'après 
(11), on peut alors exprimer $u_{1}, u_{2}, W$ en fonction des premiers termes de la somme sur $n$ :

$$
\begin{aligned}
& u_{1}=K \cos \omega_{0} t-(L-1) \sin \omega_{0} t \\
& u_{2}=K \sin \omega_{0} t+(L-1) \cos \omega_{0} t \\
& W=\omega_{0} K^{2}+K \dot{L}-(L-1) \dot{K}+(L-1)^{2} \omega_{0}^{2}
\end{aligned}
$$

avec

$K=1+\left(C_{2}+C_{-2}\right) \cos \Omega t+\left(C_{4}+C_{-4}\right) \cos 2 \Omega t+\cdots$

$L=1+\left(C_{2}-C_{-2}\right) \sin \Omega t+\left(C_{4}-C_{-4}\right) \sin 2 \Omega t+\cdots$

L'ensemble des relations (6), (9) et (12) définit le mouvement de la particule. Celui-ci se compose, en $r$ comme en $z$, d'une oscillation à la fréquence fondamentale $\omega_{0}$, modulée en amplitude par une oscillation de fréquence $\Omega$, le taux de modulation étant approximativement égal à $q / 2$.

On peut aussi chercher à exprimer le mouvement en fonction des données initiales de position et de vitesse, par exemple sous la forme :

$$
\begin{aligned}
& u=m_{11} u_{0}+m_{12} \dot{u}_{0} \\
& \dot{u}=m_{21} u_{0}+m_{22} \dot{u}_{0}
\end{aligned}
$$

soit

$$
\left(\begin{array}{l}
u \\
\dot{u}
\end{array}\right)=\left[M\left(t, \varphi_{0}\right)\right]\left(\begin{array}{c}
u_{0} \\
\dot{u}_{0}
\end{array}\right) .
$$

Nous avions par ailleurs :

$$
\begin{aligned}
& u=\alpha_{1} u_{1}(t)+\alpha_{2} u_{2}(t) \\
& \dot{u}=\alpha_{1} \dot{u}_{1}(t)+\alpha_{2} \dot{u}_{2}(t)
\end{aligned}
$$

ou encore, sous forme matricielle :

$$
\left(\begin{array}{l}
u \\
\dot{u}
\end{array}\right)=[A]\left(\begin{array}{l}
\alpha_{1} \\
\alpha_{2}
\end{array}\right)
$$

et pour la phase $\varphi_{0}$ :

$$
\left(\begin{array}{l}
u_{0} \\
\dot{u}_{0}
\end{array}\right)=\left[A_{0}\right]\left(\begin{array}{l}
\alpha_{1} \\
\alpha_{2}
\end{array}\right)
$$

avec

$$
\left[A_{0}\right]=\left|\begin{array}{ll}
u_{1}\left(\varphi_{0}\right) & u_{2}\left(\varphi_{0}\right) \\
\dot{u}_{1}\left(\varphi_{0}\right) & \dot{u}_{2}\left(\varphi_{0}\right)
\end{array}\right|
$$

En portant (14) et (15) dans (13), il vient :

$$
\begin{aligned}
& {\left[M\left(t, \varphi_{0}\right)\right]=\frac{1}{W}\left|\begin{array}{ll}
u_{1} & u_{2} \\
\dot{u}_{1} & \dot{u}_{2}
\end{array}\right|\left|\begin{array}{rr}
\dot{u}_{2}\left(\varphi_{0}\right) & -u_{2}\left(\varphi_{0}\right) \\
-\dot{u}_{1}\left(\varphi_{0}\right) & u_{1}\left(\varphi_{0}\right)
\end{array}\right|} \\
& {\left[M\left(t, \varphi_{0}\right)\right]=\frac{1}{W}[A(t)]\left[B\left(\varphi_{0}\right)\right] .}
\end{aligned}
$$

La matrice $\left[B\left(\varphi_{0}\right)\right]$ dépend uniquement de la phase $\varphi_{0}$ de création de la particule. Le temps $t$ est compté à partir de $\varphi=0$. Si l'on veut prendre comme origine des temps celle correspondant à la création et au changement d'état de la particule, il convient d'effectuer un changement de variable

$$
t=t_{1}+\varphi_{0} / \Omega
$$

où $t_{1}$ est le temps mesuré à partir de l'instant $t_{0}$ correspondant à $\varphi_{0}$.

Comme nous l'avons vu, les équations du mouvement sont linéaires, du $2^{\mathrm{e}}$ ordre, sans terme en dérivée première. Le déterminant de (16) doit donc être égal à 1 :

$$
\operatorname{det}[M]=\frac{1}{W^{2}} \operatorname{det}[A(t)] \operatorname{det}\left[B\left(\varphi_{0}\right)\right]=1 .
$$

Or, les expressions obtenues pour $W$ et $\left[B\left(\varphi_{0}\right)\right]$ donnent :

$$
\operatorname{det}\left[B\left(\varphi_{0}\right)\right]=W\left(\varphi_{0}\right)
$$

d'où

$$
\operatorname{det}[A(t)]=\operatorname{det}\left[B\left(\varphi_{0}\right)\right]=W\left(\varphi_{0}\right) .
$$

L'intérêt de la forme matricielle réside dans la rapidité et la précision de l'évaluation de la position et de la vitesse à tout instant $t$. Les trajectoires de la figure 4 ont été calculées de cette façon. On y voit clairement l'effet de modulation d'amplitude mentionné ci-dessus.

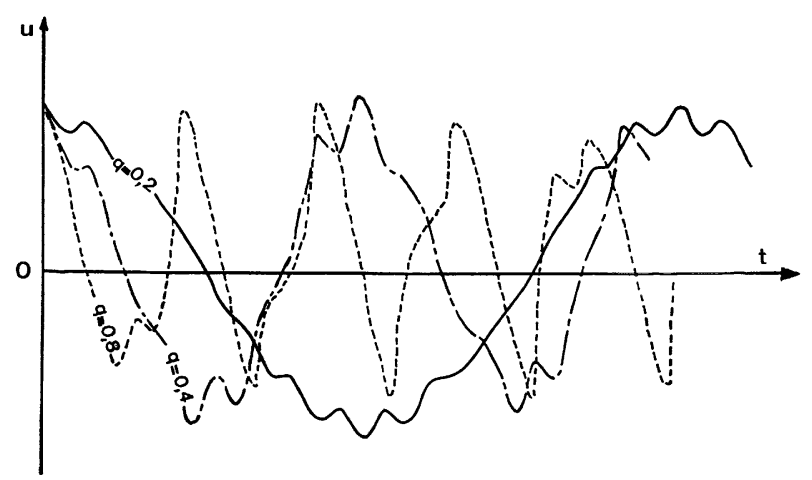

Fig. 4. - Trajectoires ioniques en fonction du temps.

2.4 Confinement et valeur initiales. - Pour qu'une particule soit confinée, il faut d'une part que son point représentatif dans le diagramme $(a, q)$ soit situé dans la zone stable, d'autre part que l'amplitude de son mouvement reste toujours inférieure aux dimensions géométriques de la cage quadrupolaire. Nous montrons ici que la valeur maximale de cette amplitude peut être reliée aux valeurs initiales $u_{0}, \dot{u}_{0}$ et $\varphi_{0}$. On peut la déduire de l'expression (6) :

$$
\left|u_{\max }\right|=\left(\alpha_{1}^{2}+\alpha_{2}^{2}\right)^{1 / 2} \sum_{-\infty}^{\infty}\left|C_{2 n}\right|
$$

En y portant les valeurs de $\alpha_{1}$ et $\alpha_{2}$ données par (9), on trouve une expression de la forme :

$$
E_{0}=C_{0} u_{0}^{2}+2 A_{0} u_{0} \dot{u}_{0}+B_{0} \dot{u}_{0}^{2}
$$


avec :

$$
\begin{aligned}
& C_{0}=\frac{\dot{u}_{10}^{2}+\dot{u}_{20}^{2}}{W} \\
& A_{0}=-\frac{u_{10} \dot{u}_{10}+u_{20} \dot{u}_{20}}{W} \\
& B_{0}=\frac{u_{10}^{2}+u_{20}^{2}}{W} \\
& E_{0}=\frac{u_{\max }^{2} W}{\left(\sum_{n}\left|C_{2 n}\right|\right)^{2}} .
\end{aligned}
$$

Pour alléger l'écriture, nous avons posé ici :

$$
\begin{aligned}
u_{i 0} & =u_{i}\left(\varphi_{0}\right) \\
\dot{u}_{i 0} & =\dot{u}_{i}\left(\varphi_{0}\right) \\
i & =1 \text { ou } 2 .
\end{aligned}
$$

Pour $a, q$ et $\varphi_{0}$ donnés, l'amplitude maximale $u_{\max }$ sera la même pour toutes les particules dont les conditions initiales vérifient l'éq. (18).

L'éq. (18) est celle d'une ellipse, de surface égale à $S=\pi E_{0}$. Pour $a$ et $q$ donnés, l'orientation et l'excentricité de l'ellipse varient avec $\varphi_{0}$, phase de création de la particule. La figure 5 donne quelques points caractéristiques où $u_{0}$ et $\dot{u}_{0}$ sont, soit nuls, soit maximums.

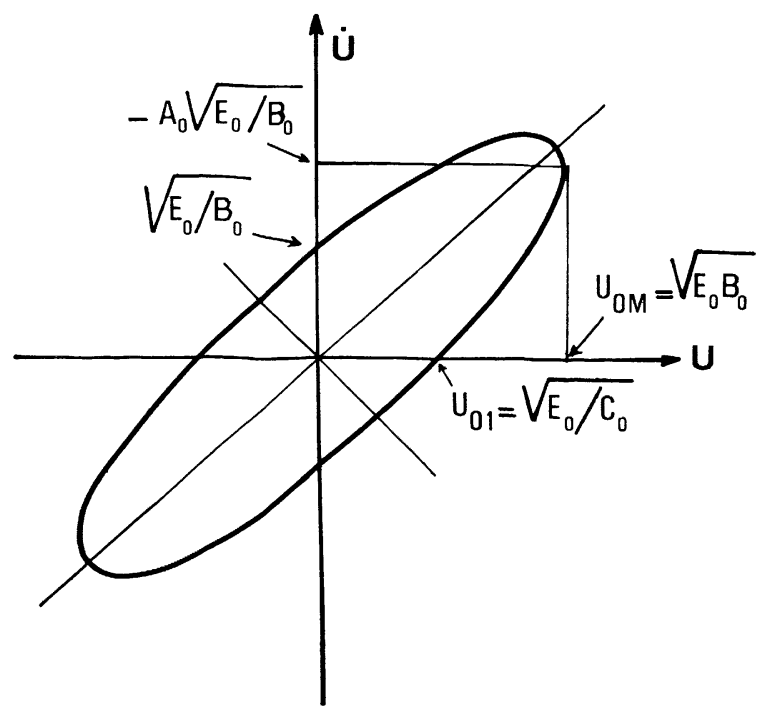

Fig. 5. - Points caractéristiques de l'ellipse de l'espace de phase.

En posant $u_{\max }=r_{0}$ ou $z_{0}$, dimension minimale du piège, toute particule représentée à la phase $\varphi_{0}$ par un couple de points $\left(u_{0}, \dot{u}_{0}\right)$ situés à l'intérieur de l'ellipse correspondante, sera confinée. Appliquons au cas particulier d'un ion de vitesse initiale négligeable ; ce sera le cas, en première approximation, d'un ion monochargé dont la formation à partir d'un atome neutre s'est effectuée à l'instant correspondant à la phase $\varphi_{0}$ du champ HF. Considérons le mouvement en $z$ et posons $u_{\max }=z_{0}$. La position initiale $u_{0 \mathrm{M}}$ à laquelle pourra se trouver l'ion pour être confiné (c'est-à-dire pour lequel l'amplitude maximum de l'oscillation suivant $O z$ est égale à $u_{\max }$ ) sera telle que (Fig. 5) :

$$
\begin{aligned}
u_{0 \mathrm{M}} & =\left(E_{0} / C_{0}\right)^{1 / 2} \\
& =\frac{u_{\max } \sqrt{W}}{\sum\left|C_{2 n}\right|} \frac{\sqrt{W}}{\left(\dot{u}_{10}^{2}+\dot{u}_{20}^{2}\right)^{1 / 2}} .
\end{aligned}
$$

Introduisons la quantité

$$
R_{z}=\frac{u_{0 \mathrm{M}}}{z_{0}}
$$

qui représente le rendement du piège, c'est-à-dire le rapport du nombre d'ions confinés au nombre d'ions créés. La figure 6 donne $R_{z}$ en fonction de $\varphi_{0}$ pour différentes valeurs de $q$.

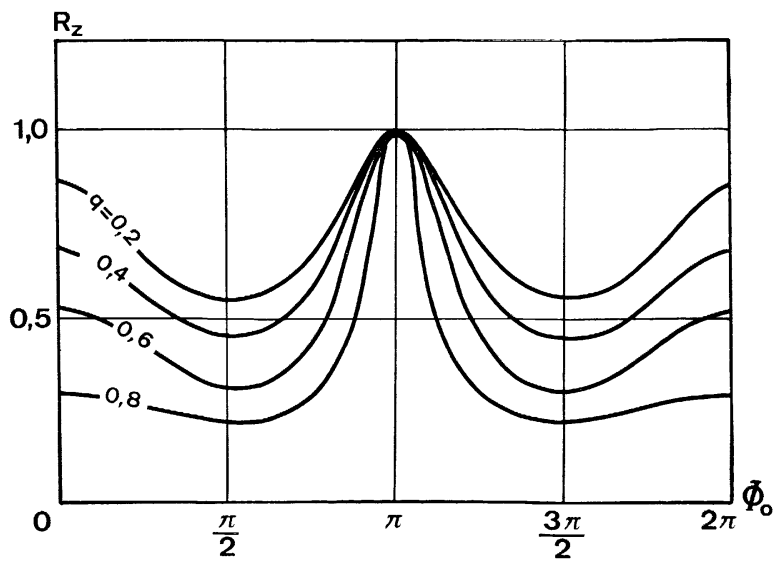

Fig. 6. - Rendement du piège en fonction de la phase HF, pour le passage $Z=0$ à $Z=1$.

Sur une période du champ HF, le rendement est :

$$
R_{z(2 \pi)}=\frac{1}{2 \pi} \int_{0}^{2 \pi} \frac{u_{0 M}\left(\varphi_{0}\right)}{z_{0}} \mathrm{~d} \varphi_{0}=f(q) .
$$

On pourrait de même étudier le mouvement en $r$, avec cependant une simplification dans le cas où le faisceau ionisant se propage suivant $O z$; la position initiale maximale est alors limitée au rayon du faisceau. Le calcul des trajectoires montre que dans ces conditions, le déplacement maximal ne dépasse pas trois fois la valeur de $u_{\mathrm{OM} r}$. L'amplitude en $r$ restant toujours inférieure à la dimension limite $r_{0}$ du piège, le rendement total ne sera pas affecté par le mouvement en $r$.

2.5 PASSAgE de $Z=n$ A $Z=n+1$. Rendement DU PIÈGE. - Etudions maintenant le processus de passage aux états de charge supérieurs à un. Soit une particule de charge $n$, de paramètres $a_{n}$ et $q_{n}$, confinée dans la cage. L'ionisation, qui correspond au passage à l'état de charge $(n+1)$, surviendra lors d'un choc de 
la particule avec un électron du faisceau, à l'instant correspondant à la phase $\varphi_{0 n}$. Les conditions initiales pour la nouvelle particule seront $u_{0 n}$ et $\dot{u}_{O n}$, valeurs prises à l'instant du choc; contrairement à la vitesse initiale $\dot{u}_{0}$ de l'ion de charge unité, $\dot{u}_{0 n}$ pourra prendre des valeurs élevées. Si le point

$$
\left(a_{n+1}=a_{n} \cdot \frac{n+1}{n}, q_{n+1}=q_{n} \cdot \frac{n+1}{n}\right)
$$

représentatif du nouvel état appartient aussi à la zone de stabilité du diagramme $(a, q)$, la nouvelle particule aura une trajectoire stable. Elle sera confinée si de plus le couple $\left(u_{0 n}, \dot{u}_{0 n}\right)$ appartient à une ellipse déterminée par $\left(a_{n+1}, q_{n+1}\right)$ et $\varphi_{0 n}$, ellipse définissant les valeurs initiales maximales à ne pas dépasser.

Afin d'augmenter le rendement - rapport des ions confinés aux ions créés - il est utile de connaître les phases les plus défavorables pour lesquelles les conditions initiales seront les plus restrictives. Théoriquement, on peut imposer que l'ionisation se produise en dehors de ces phases, par exemple en hachant le faisceau par des impulsions de tension appliquées au canon et synchronisées avec le champ HF de confinement.

M. Baril et A. Septier [5] ont montré que des points d'une trajectoire stable, choisis de façon à avoir la même phase (à $2 k \pi$ près) par rapport au champ HF, appartiennent à une même ellipse dans l'espace des phases $(u, \dot{u})$ (Fig. 7) :

$$
C u^{2}+2 A u \dot{u}+B \dot{u}^{2}=E .
$$

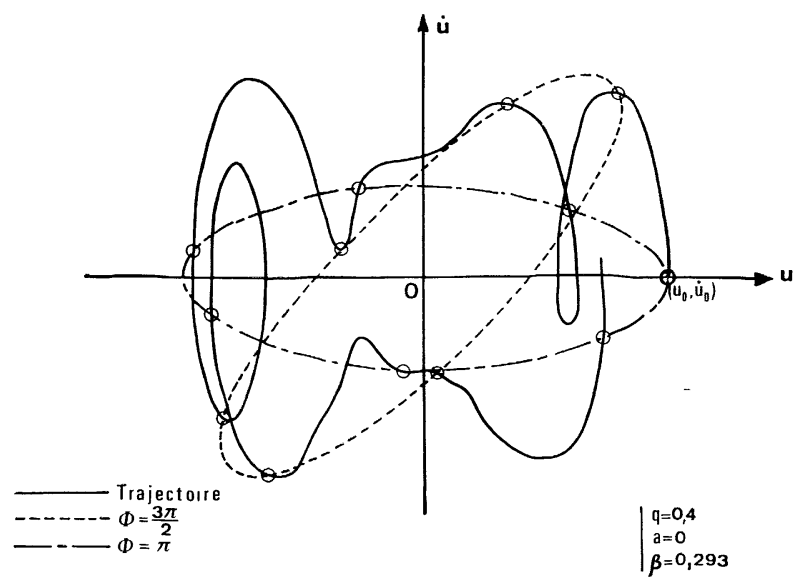

Fig. 7. - Trajectoires ioniques dans l'espace de phase.

Ils ont déterminé la matrice $\mathcal{H}(\varphi)$ qui permet de passer du point $\{u, \dot{u}(\varphi+2 k \pi)\}$ au point

$$
\{u, \dot{u}[\varphi+(2 k+1) \pi]\} \text {. }
$$

Les éléments de cette matrice permettent, pour une phase $\varphi$ donnée, de calculer les paramètres $A, B, C$ qui vont servir dans la suite.

A la phase $\varphi_{\text {on }}$ du champ HF, juste avant le choc ionisant, l'état de charge $n$ est donc défini par l'ellipse sur laquelle se trouve dans l'espace des phases le point représentatif de l'ion ayant comme amplitudes maximales celles permises par les dimensions du piège. Cet état de charge est donc caractérisé par :

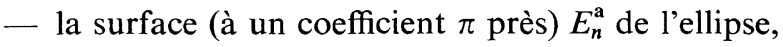
que l'on peut appeler par extension acceptance du piège pour la charge $n$;

- les paramètres $A_{n}, B_{n}, C_{n}$ de cette ellipse, déterminés par les valeurs de $a_{n}, q_{n}$ et $\varphi_{0 n}$.

$\mathrm{Au}$ même instant, mais juste après l'ionisation, le nouvel état de charge $(n+1)$ sera figuré par l'ellipse $\left(A_{n+1}, B_{n+1}, C_{n+1}, E_{n+1}^{\mathrm{e}}\right)$ circonscrite à l'ellipse précédente (Fig. 8). Cette ellipse représente pour la phase $\varphi_{0 n}$ l'enveloppe des nouvelles trajectoires ioniques correspondant à l'ensemble des valeurs initiales représentées par l'ellipse $\left(A_{n}, B_{n}, C_{n}, E_{n}^{\mathrm{a}}\right)$. On est donc sûr qu'à toute valeur initiale appartenant à cette dernière correspondra, pour le nouvel état de charge, une ellipse homothétique de l'ellipse

$$
\left(A_{n+1}, B_{n+1}, C_{n+1}, E_{n+1}^{\mathrm{e}}\right)
$$

dans un rapport inférieur à un.

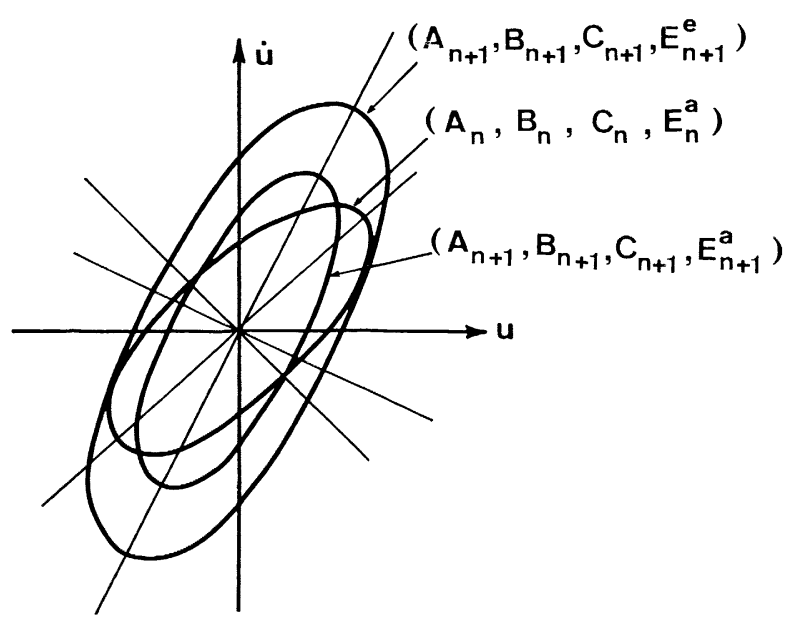

Fig. 8. - Représentation dans l'espace de phase du passage

$$
Z=n \text { à } Z=n+1 \text {. }
$$

L'ellipse $\left(A_{n+1}, B_{n+1}, C_{n+1}, E_{n+1}^{\mathrm{e}}\right)$ sera définie par : - les paramètres $A_{n+1}, B_{n+1}, C_{n+1}$ déterminés par les coefficients $a_{n+1}, q_{n+1}$ et $\varphi_{0 n}$;

- la surface $E_{n+1}^{\mathrm{e}}$ que l'on peut appeler émittance du nuage ionique pour la charge $(n+1)$; en fonction de $E_{n}^{a}$, elle s'exprime par la relation [6] :

$$
E_{n+1}^{\mathrm{e}}=E_{n}^{\mathrm{a}}\left(D+\sqrt{D^{2}-1}\right)
$$

avec

$$
D=\frac{1}{2}\left(B_{n} C_{n+1}+C_{n} B_{n+1}-2 A_{n} A_{n+1}\right) .
$$

L'acceptance $E_{n+1}^{\mathrm{a}}$ du piège pour la charge $(n+1)$ n'est autre que l'ellipse homothétique de l'ellipse $\left(E_{n+1}^{\mathbf{e}}\right)$, 
dont la surface est déterminée par la limitation des amplitudes des oscillations aux dimensions du piège.

Déterminons l'acceptance $E_{n+1}^{\mathrm{a}}$. Nous avons vu (Fig. 5) que le déplacement maximal est égal à :

$$
u_{\max }=\sqrt{E B} .
$$

Appliquons au mouvement en $z ; u_{\max }=z_{0}$, d'où

$$
E_{n+1}^{\mathrm{a}}=\frac{z_{0}^{2}}{\left(B_{n+1}\right)_{\max }} .
$$

En définissant maintenant le rendement du piège comme le rapport de l'acceptance sur l'émittance pour un même état de charge $(n+1)$, nous avons

$$
R_{n \rightarrow n+1}\left(\varphi_{0 n}\right)=\frac{E_{n+1}^{\mathrm{a}}}{E_{n+1}^{\mathrm{e}}}=\frac{z_{0}^{2}}{E_{n+1}^{\mathrm{e}}\left(B_{n+1}\right)_{\max }} .
$$

Par ailleurs, l'acceptance du piège pour la charge $n$ peut s'écrire :

$$
E_{n}^{\mathrm{a}}=\frac{z_{0}^{2}}{\left(B_{n}\right)_{\max }}
$$

d'où

$$
E_{n+1}^{\mathrm{e}}=\frac{z_{0}^{2}}{\left(B_{n}\right)_{\max }}\left(D+\sqrt{D^{2}-1}\right)
$$

et le rendement prend donc la forme :

$$
R_{n \rightarrow n+1}\left(\varphi_{0 n}\right)=F\left(\varphi_{0 n}\right) \frac{\left(B_{n}\right)_{\max }}{\left(B_{n+1}\right)_{\max }}
$$

avec

$$
F\left(\varphi_{0 n}\right)=D-\sqrt{D^{2}-1}
$$

Nous avons calculé numériquement la variation de $F\left(\varphi_{0 n}\right)$ pour différentes valeurs de $q$ et pour le cas du passage de $Z=1$ à $Z=2$. Les courbes obtenues ont la même allure que celles de la figure 6 qui donnent le rendement pour le passage $Z=0$ à $Z=1$. La grandeur $F$ est maximum pour $\varphi_{0}=(2 k+1) \pi$ et de plus, quand $q$ tend vers zéro, les phases $\varphi_{0}=2 k \pi$ viennent s'ajouter aux phases favorables. Les phases défavorables pour le mouvement en $r$ et en $z$ coïncident, puisque changer $q$ en $-q$ revient à changer la phase de $\pi$. Ces résultats sont valables pour $n$ quelconque et permettent de déterminer les conditions expérimentales optimales pour l'obtention du meilleur rendement final.

2.6 EfFETS DE LA CHARGE D'ESPACE ÉLECTRONIQUe. - La charge d'espace électronique est en elle-même un moyen de confinement des ions. Au moment de leur création dans le faisceau, les ions subissent l'effet du champ électrique dû aux électrons et sont attirés vers l'axe de révolution du système. La population électronique comprend les électrons rapides du faisceau et les électrons lents provenant des collisions ionisantes; dans les conditions expérimentales habituelles ces derniers ont un rapport $(e / m)$ tel qu'ils sont instables dans le champ HF et sont donc très vite éliminés.
Le champ électrique radial est donné par le théorème de Gauss :

$$
E_{r}=\frac{\rho_{r}}{2 \varepsilon_{0}}
$$

avec

$$
\rho=\rho_{\mathrm{i}}-\rho_{\mathrm{e}}
$$

$\rho_{\mathbf{i}}=$ densité de charge ionique,

$\rho_{\mathrm{e}}=$ densité de charge électronique

$$
\rho_{\mathrm{e}}=\frac{I_{\mathrm{f}}}{S\left(2 \mathrm{e} V_{\mathrm{f}} / m\right)^{1 / 2}}
$$

$I_{\mathrm{f}}=$ courant du faisceau électronique ;

$V_{\mathrm{f}}=$ tension du faisceau électronique;

$S=$ section du faisceau électronique.

Dans le cas de la création des ions monochargés dont la vitesse initiale est la vitesse thermique des atomes, le confinement dû à la charge d'espace électronique négative existera jusqu'à la neutralisation totale de celle-ci, c'est-à-dire jusqu'au temps $\tau_{N_{i}=N_{0}}$ tel que :

$$
N_{\mathrm{i}}=N_{0} N_{\mathrm{e}}<\sigma_{0-1} v>t
$$

d'où

$$
\tau_{N_{\mathbf{i}}=N_{\mathrm{e}}}=\frac{1}{N_{0}<\sigma_{0-1} v>}
$$

avec $N_{0}=$ densité de neutres ;

$\sigma_{0-1}=$ section efficace d'ionisation de l'état neutre à l'état $Z=1$.

Pour un faisceau électronique de $500 \mathrm{~V}-10 \mu \mathrm{A}$, de $2 \mathrm{~mm}$ de diamètre, tombant sur un gaz d'argon à la pression de $2 \times 10^{-8}$ torr, on a :

$$
\begin{gathered}
N_{\mathrm{e}} \approx 1,5 \times 10^{12} / \mathrm{m}^{3} ; \quad N_{0} \approx 7 \times 10^{14} / \mathrm{m}^{3} ; \\
\sigma_{0-1} \approx 1 \times 10^{-16} \mathrm{~cm}^{2}
\end{gathered}
$$

d'où

$$
\tau_{N_{\mathrm{i}}=N_{\mathrm{e}}} \approx 1 \times 10^{-2} \mathrm{~s} .
$$

Une fois la neutralisation achevée, les nouveaux ions créés seront confinés par le champ quadrupolaire seul. Une nouvelle saturation de charge surviendra lorsque les effets divergents de la charge d'espace due à l'accumulation des ions ne seront plus contrebalancés par le champ quadrupolaire.

La charge d'espace électronique a aussi pour effet de défocaliser le faisceau ; cet inconvénient est limité par l'emploi d'un champ magnétique, lequel, comme on le verra plus loin, aura aussi une influence sur le confinement.

2.7 EfFets de la Charge D'espaCe iOniQue. — La quantité maximum d'ions confinés par le champ quadrupolaire est finalement limitée par les effets de charge d'espace ionique. Sa valeur maximale sera atteinte quand la force de confinement due au champ 
HF sera contrebalancée dans l'une des directions $O r$ ou $O z$ par la force exercée par le champ de charge d'espace ionique.

Calculons d'abord la force moyenne exercée par le champ HF pendant une période de ce champ. L'équation du mouvement d'une particule dans un champ électrique, est :

$$
M \frac{\mathrm{d}^{2} \mathbf{u}}{\mathrm{d} t^{2}}=Z e \mathbf{E}_{0}(u) \cos \Omega t .
$$

Dans le cas du champ HF dont la fréquence $\Omega$ est supérieure à la fréquence d'oscillation $\omega$ de la particule, la solution de (20) peut se décomposer en deux termes [7] :

- un terme $u_{0}(t)$ de fréquence $\omega$;

— un terme $\zeta$ de faible amplitude et de fréquence $\Omega$.

Développons (20) par rapport à $\zeta$ :

$$
\begin{aligned}
M \frac{\mathrm{d}^{2} \mathbf{u}_{0}(t)}{\mathrm{d} t^{2}} & +M \frac{\mathrm{d}^{2} \zeta}{\mathrm{d} t^{2}}= \\
& =Z e\left[\mathbf{E}_{0}\left(u_{0}\right) \cos \Omega t+\zeta\left(\frac{\partial \mathbf{E}_{0}}{\partial \mathbf{u}}\right)_{u_{0}} \cos \Omega t\right] .
\end{aligned}
$$

Identifions les termes de même fréquence et d'amplitude du même ordre :

$$
\begin{gathered}
M \frac{\mathrm{d}^{2} \zeta}{\mathrm{d} t^{2}}=Z e \mathbf{E}_{0}\left(u_{0}\right) \cos \Omega t \\
M \frac{\mathrm{d}^{2} \mathbf{u}_{0}(t)}{\mathrm{d} t^{2}}=Z e \zeta\left(\frac{\partial \mathbf{E}_{0}}{\partial \mathbf{u}}\right)_{u_{0}} \cos \Omega t .
\end{gathered}
$$

En prenant pour $\zeta$ la forme définie plus haut, on en déduit :

$$
\zeta=-\frac{Z e}{M} \frac{\mathbf{E}_{0}\left(u_{0}\right)}{\Omega^{2}} \cos \Omega t .
$$

Moyennons l'éq. (21) sur une période de champ HF, en y remplaçant $\zeta$ par son expression donnée par (22). On obtient :

$$
\overline{M \frac{\mathrm{d}^{2} \mathbf{u}_{0}(t)}{\mathrm{d} t^{2}}}=-\frac{(Z e)^{2}}{4 M \Omega^{2}} \frac{\partial \mathbf{E}_{0}^{2}\left(u_{0}\right)}{\partial u} .
$$

La force moyenne agissant sur une particule dérive donc d'un potentiel $\varphi$ :

$$
\overline{\mathbf{F}\left(u_{0}\right)}=-Z e \nabla \varphi
$$

dont la valeur est :

$$
\varphi=\frac{Z e}{4 M \Omega^{2}} E_{0}^{2}\left(u_{0}\right) .
$$

D'après la forme du champ quadrupolaire (voir éq. (2)), on a pour $\varphi$ l'expression suivante :

$$
\varphi=\frac{Z e}{4 M \Omega^{2}} \frac{V^{2}}{r_{0}^{4}}\left(r^{2}+4 z^{2}\right) .
$$

Par ailleurs, la force due à la charge d'espace ionique vaut :

$$
\mathbf{F}_{\mathrm{i}}=-Z e \nabla \varphi_{\mathrm{i}}
$$

avec

$$
\Delta \varphi=-\frac{\rho_{\mathrm{i}}}{\varepsilon_{0}} .
$$

La charge maximale pouvant être piégée est, en première approximation, celle pour laquelle on a la relation :

$$
\mathbf{F}_{\mathbf{i}}=-\overline{\mathbf{F}\left(u_{0}\right)} .
$$

En prenant le gradient de (24)

$$
-\Delta \varphi=\Delta \varphi_{\mathrm{i}}=-\frac{\rho_{\mathrm{i}-\max }}{\varepsilon_{0}} .
$$

On en déduit, avec (23) :

$$
\rho_{\mathrm{i}-\max }=\frac{3}{4} \frac{\varepsilon_{0} Z e V^{2}}{M \Omega^{2} z_{0}^{4}}
$$

ou encore, avec $\rho_{\mathrm{i}-\max }=Z e N_{\mathrm{i}-\max }$ :

$$
N_{\text {i-max }}=\frac{3}{16} \varepsilon_{0} M\left(\frac{\Omega q_{z}}{Z e}\right)^{2} .
$$

Le potentiel de charge d'espace $\varphi_{\mathrm{i}}$ peut s'écrire, en utilisant (23) et (25)

$$
\varphi_{\mathrm{i}}=-\frac{\rho_{\mathrm{i}}}{12 \varepsilon_{0}}\left(r^{2}+4 z^{2}\right) .
$$

Les équipotentielles sont donc des ellipses homothétiques. La différence de potentiel existant entre l'équipotentielle passant par le point $\left(r_{0}, 0\right)$ et celle passant par le point $\left(0, z_{0}\right)$ est :

$$
\Delta V_{\mathrm{i}}=\varphi_{\mathrm{i}}\left(r_{0}, 0\right)-\varphi_{\mathrm{i}}\left(0, z_{0}\right)=\frac{\rho_{\mathrm{i}} z_{0}^{2}}{6 \varepsilon_{0}}
$$

La charge d'espace ionique équivaut donc phénoménologiquement à une tension continue $\Delta V_{\mathrm{i}}$ appliquée entre les chapeaux et l'électrode centrale de la cage quadrupolaire. A cette tension, qui exerce un effet défocalisant sur les ions, correspondent des variations du paramètre $a$.

Du potentiel $\varphi_{\mathrm{i}}$ dérivent les champs :

$$
\begin{aligned}
& E_{\mathrm{ir}}=\frac{\rho_{\mathrm{i}}}{6 \varepsilon_{0}} r \\
& E_{\mathrm{i} z}=\frac{2 \rho_{\mathrm{i}}}{3 \varepsilon_{0}} z .
\end{aligned}
$$

On remarquera que, contrairement aux champs quadrupolaires, les champs de charge d'espace ionique sont de même signe, car les forces défocalisantes exercées sur les ions sont de même sens en $r$ et en $z$. Injectés dans les équations du mouvement, les champs $E_{\mathrm{i}}$ conduisent à de nouvelles valeurs $a^{\prime}$ telles que :

$$
a^{\prime}=a+\Delta a
$$


avec :

$$
\Delta a_{r}=\frac{\Delta a_{z}}{4}=-\frac{2}{3} \frac{Z e \rho_{\mathrm{i}}}{M \varepsilon_{0} \Omega^{2}} .
$$

Comme le montre la figure 9, la charge d'espace ionique fait glisser les lignes iso- $\beta$ et la zone de stabilité s'en trouve réduite. Des particules se trouvant initialement en zone stable peuvent ainsi devenir instables quand la charge d'espace augmente. La relation (28) fournit ainsi un moyen pratique d'estimation du nombre d'ions confinés ; pour cela, il suffit de déterminer expérimentalement une ligne iso- $\beta$ puis de calculer l'écart $\Delta a$ qui la sépare de la ligne iso- $\beta$ théorique correspondante (cf. $\S 3.3$ ).

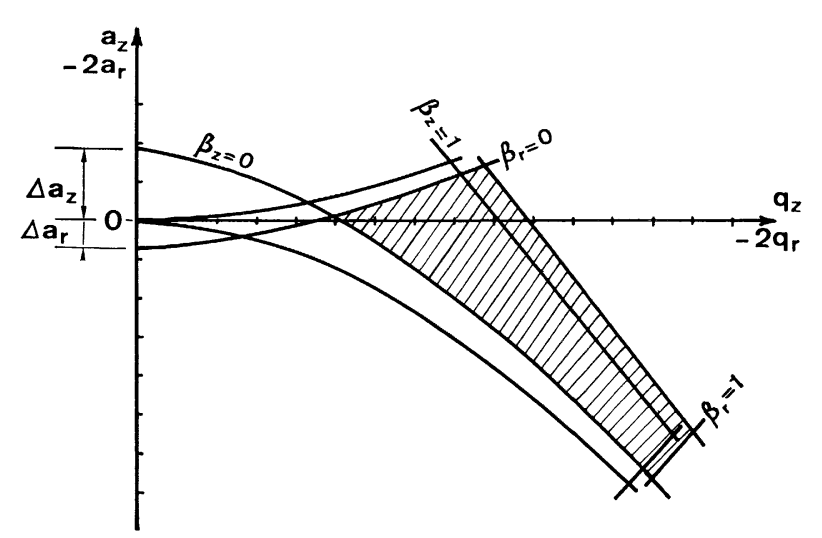

FIG. 9. - Modification du diagramme de stabilité avec la charge d'espace ionique.

Les surfaces équipotentielles déterminées par (27) sont des ellipsoïdes de révolution et le volume de confinement possède l'une de ces surfaces comme enveloppe. Le volume de confinement maximum inscrit dans l'espace inter-électrodes correspond alors à un ellipsoïde d'équation

$$
r^{2}+4 z^{2}=r_{0}^{2} \text {. }
$$

Les demi-axes $m$ et $n$ ont pour valeur :

$$
m=r_{0}, \quad n=r_{0} / 2
$$

et le volume de l'ellipsoïde est égal à :

$$
V_{\text {cft-max }}=\frac{4}{3} \pi m n^{2}=\frac{1}{3} \pi r_{0}^{3} \text {. }
$$

Le volume d'ionisation est égal au volume du faisceau d'électrons intercepté par le volume de confinement, soit :

$$
V_{\text {ion }}=\pi a_{0}^{2} .2 n=\pi a_{0}^{2} r_{0}
$$

( $a_{0}=$ rayon du faisceau).

Il en découle que :

$$
\frac{V_{\text {ion }}}{V_{\text {cft-max }}}=3\left(\frac{a_{0}}{r_{0}}\right)^{2} \text {. }
$$

On a en général $a_{0} \ll r_{0}$; le volume d'ionisation est donc très petit par rapport au volume de confinement où évoluent les ions. On doit donc s'attendre à trouver des temps d'ionisation expérimentaux supérieurs aux temps théoriques.

2.8 EFFETS DU CHAMP MAGNÉTIQUE. — Le champ magnétique statique axial a pour premier rôle de focaliser le faisceau électronique ionisant, en diminuant la divergence due à sa propre charge d'espace. Mais il agit aussi sur les ions, dont l'équation du mouvement s'écrit maintenant :

$$
M \frac{\mathrm{d}^{2} \mathbf{u}}{\mathrm{d} t^{2}}=Z e(\mathbf{E}+\mathbf{v} \wedge \mathbf{B}) .
$$

Dans le système de coordonnées $(x, y, z)$, le champ magnétique $\mathbf{B}\left(0,0, B_{z}\right)$ couple les équations en $x$ et $y$ mais laisse inchangée l'équation en $z$. Le mouvement en $z$ ne sera donc pas modifié :

$$
\begin{aligned}
& M \frac{\mathrm{d}^{2} x}{\mathrm{~d} t^{2}}=Z e\left(u_{0}-V \cos \Omega t\right) \frac{x}{z_{0}^{2}}+Z e \frac{\mathrm{d} y}{\mathrm{~d} t} B_{z} \\
& M \frac{\mathrm{d}^{2} y}{\mathrm{~d} t^{2}}=Z e\left(u_{0}-V \cos \Omega t\right) \frac{y}{z_{0}^{2}}-Z e \frac{\mathrm{d} x}{\mathrm{~d} t} B_{z} .
\end{aligned}
$$

On peut découpler les équations précédentes en faisant le changement de variables suivant

$$
\begin{aligned}
& u_{+}=x+j y \\
& u_{-}=x-j y
\end{aligned}
$$

On obtient alors

$$
M \frac{\mathrm{d}^{2} u_{ \pm}}{\mathrm{d} t^{2}}=Z e\left(u_{0}-V \cos \Omega t\right) \frac{u_{ \pm}}{z_{0}^{2}} \mp j \frac{\mathrm{d} u_{ \pm}}{\mathrm{d} t} Z e B_{z} .
$$

De telles équations admettent comme solution :

$$
u_{ \pm}=r \mathrm{e}^{ \pm j \omega_{\mathrm{L}} t}
$$

avec $\omega_{\mathrm{L}}=$ fréquence angulaire de Larmor $=\frac{1}{2} \frac{Z e B_{z}}{M}$ et où $r$ est la solution de :

$$
\frac{\mathrm{d}^{2} r}{\mathrm{~d} t^{2}}+\left(\omega_{\mathrm{L}}+\frac{a_{r} \Omega^{2}}{4}+\frac{q_{r} \Omega^{2}}{2} \cos \Omega t\right) r=0 .
$$

Si l'on se ramène à la forme des équations de Mathieu, il lui correspond la valeur $a_{r}^{\prime}$ suivante :

$$
a_{r}^{\prime}=a_{r}+\Delta a_{r}
$$

avec

$$
\Delta a_{r}=\frac{4 \omega_{\mathrm{L}}^{2}}{\Omega^{2}}
$$

Le champ magnétique statique a donc pour effet d'augmenter la zone de stabilité (Fig. 10). Cet effet est cependant très faible et le plus souvent négligeable. En effet, pour un champ de $100 \mathrm{G}$ (ou $10^{-2}$ tesla) et pour un ion $\mathrm{Ar}^{+}$, nous avons : $\omega_{\mathrm{L}} \approx 1 \times 10^{4}$. 


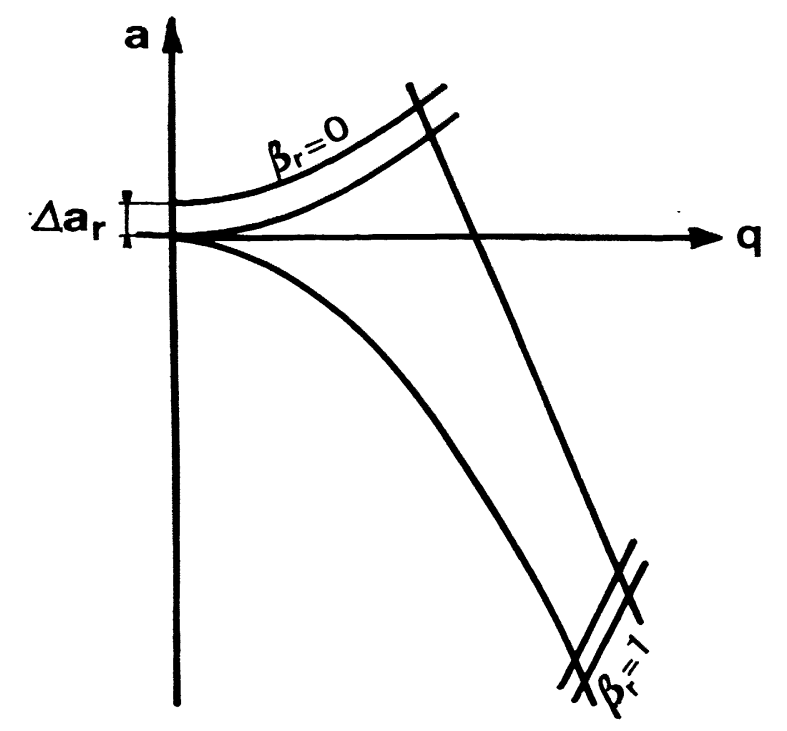

FIG. 10. - Modification du diagramme de stabilité avec le champ magnétique statique.

En prenant $\Omega=2 \pi\left(4 \times 10^{5}\right)$, nous trouvons :

$$
\Delta a_{r} \approx 6 \times 10^{-5} \text { ou encore } \frac{\Delta a_{r}}{a_{r}} \approx 10^{-4} .
$$

3. Résultats expérimentaux. - 3.1 DISPOSITIF EXPÉRIMENTAL. - Pour des raisons pratiques de réalisation, la forme hyperbolique théorique de la section méridienne des électrodes n'a pas été respectée, mais seulement approchée par une forme circulaire. La cage quadrupolaire se compose donc de deux calottes sphériques identiques, placées en $z= \pm z_{0}$, de part et d'autre d'une électrode de révolution ayant un arc de cercle pour méridienne et de rayon de gorge $r_{0}$. Les trois électrodes sont faites en grille d'acier inoxydable de $2 \mathrm{~mm}$ de pas et isolées les unes des autres par des entretoises en quartz. Les deux calottes sont percées en leur centre d'un trou de $6 \mathrm{~mm}$ de diamètre pour le passage du faisceau électronique. Les dimensions caractéristiques sont :

$$
r_{0}=35 \mathrm{~mm}, \quad z_{0}=25 \mathrm{~mm}\left(r_{0} \approx z_{0} \sqrt{2}\right) .
$$

Le canon à électrons est du type triode et peut émettre jusqu'à $500 \mathrm{~mA}$ sous $6 \mathrm{kV}$. Un diaphragme de $3 \mathrm{~mm}$ de diamètre limite les dimensions du faisceau avant son entrée dans la cage. La focalisation du faisceau est assurée par un champ magnétique d'une valeur maximum de $400 \mathrm{G}$, créé par deux bobines plates en position de Helmholtz. L'ensemble canoncage-collecteur est placé dans une enceinte dont le vide limite est de $2 \times 10^{-10}$ torr avant introduction du gaz à ioniser.

3.2 Schéma électrique. Détection. - Plusieurs états de charge pouvant exister en même temps dans le piège, il est nécessaire d'utiliser une méthode de détection qui puisse les discriminer. Nous avons vu que les ions confinés dans le champ HF oscillent en $r$ et en $z$ à des fréquences $\omega_{r}$ et $\omega_{z}$ qui dépendent de leur état de charge. C'est cette caractéristique que nous allons utiliser pour la détection des différentes charges piégées.

Le montage électrique est schématisé sur la figure 12. La tension $V$ de confinement, de fréquence $F$, est appliquée en série avec la tension continue $U_{0}$ entre l'électrode centrale et les chapeaux. Par ailleurs, l'ensemble de la cage est polarisé par la tension $U_{\mathrm{p}}$, afin d'éviter que les électrodes, qui ne sont pas toutes reliées directement à la masse, ne soient chargées par les électrons lents captés.

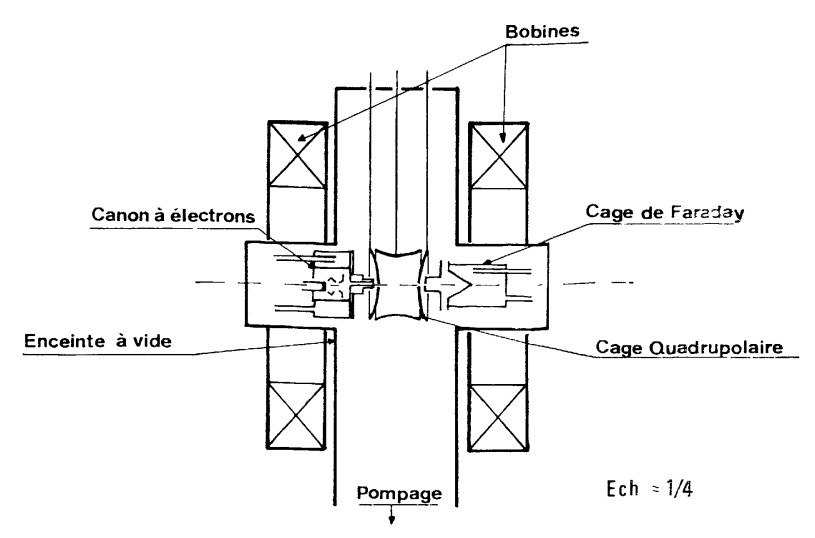

Fig. 11. - Schéma du montage expérimental.

Les chapeaux de la cage sont branchés aux bornes d'un circuit L-C et l'ensemble forme un circuit oscillant de fréquence propre $f_{0}$. Ce circuit est relié à un générateur de fréquence $f_{z}$ à travers une résistance $R$ de grande valeur. La tension $V_{\mathrm{d}}$ recueillie aux bornes de (L-C) est amplifiée puis détectée par une détection de phase. En maintenant $f_{z}=f_{0}=$ Cte, nous faisons varier l'une des tensions de confinement, $U_{0}$ ou $V$, ce qui entraîne la variation des paramètres $a$ ou $q$, c'est-à-dire la variation de la fréquence d'oscillation ionique $f_{\mathrm{i}}$ (éq. (10)). Quand $f_{\mathrm{i}}$ devient égale à $f_{z}$, le mouvement ionique entre en résonance avec le champ à la fréquence $f_{z}$ et les ions acquièrent de l'énergie aux dépens du champ HF. Il s'ensuit un amortissement du circuit L-C et une diminution de la tension $V_{\mathrm{d}}$ aux bornes. Seule la composante en $z$ des oscillations ioniques intervient ici, car la tension de détection à $f_{z}$ est appliquée entre les deux chapeaux, suivant l'axe des $z$.

Sur la figure 12 nous avons représenté des filtres passifs et actifs, dont l'emploi a été rendu nécessaire pour éliminer les effets du rayonnement parasite provenant du signal HF de confinement.

3.3 RÉSUltats. - Les principaux paramètres utilisés dans nos expériences ont pour valeurs :

$F$, fréquence du champ $\mathrm{HF}$ de confinement $=400 \mathrm{kHz}$;

$V$, tension $\mathrm{HF}$ de confinement $=0$ à $2000 \mathrm{~V}$; 


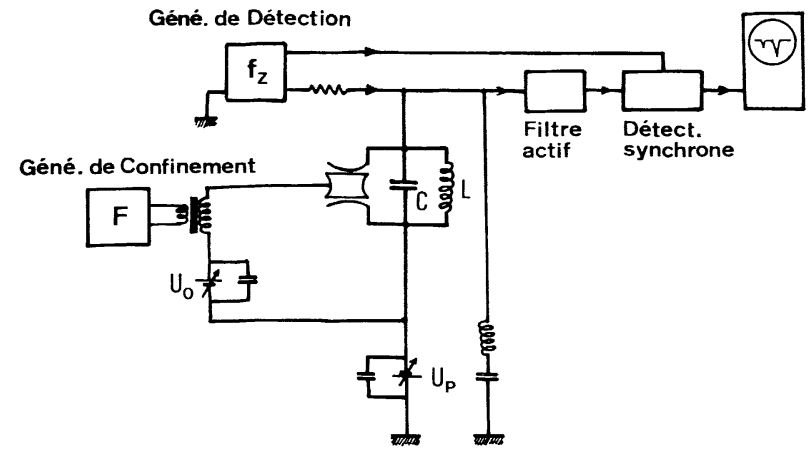

Fig. 12. - Schéma du montage électrique.

$f_{z}$, fréquence de détection $=50$ à $100 \mathrm{kHz}$, ce qui donne un $\beta_{z}$ correspondant de 0,25 à 0,50 ;

$v_{z}$, tension à $f_{z}$ appliquée aux chapeaux de la cage : quelques $\mathrm{mV}$.

Le gaz utilisé a été principalement de l'argon, à une pression comprise entre $10^{-8}$ et $10^{-7}$ torr (densité $N_{0} \approx 10^{15} / \mathrm{m}^{3}$ ).

La sensibilité du montage de détection a été testée en travaillant à la plus basse pression possible et avec des courants de faisceau très faibles. Pour un faisceau de $50 \mathrm{~V}-3 \mu \mathrm{A}$, des ions argon de charge $Z=1$ ont pu être détectés avec un rapport signal sur bruit égal à cinq, pour une pression de $4,6 \times 10^{-9}$ torr.

Connaissant la courbe $\beta_{z}$ théorique imposée par la fréquence de détection $\left(\beta_{z}=2 f_{z} / F\right)$, nous avons ensuite déterminé point par point la courbe expérimentale correspondante. Pour cela, nous pouvons, soit faire varier $V$ et réajuster $U_{0}$, soit faire l'inverse, de façon à toujours ramener les oscillations à la fréquence $f_{z}$. Chaque couple de tensions $\left(V-U_{0}\right)$ correspond à un point $(a, q)$ du diagramme de stabilité. Ainsi, pour :

$$
p=1,5 \times 10^{-8} \text { torr }, f_{z}=65 \mathrm{kHz},
$$

soit

$$
\beta_{z \text { (théor.) }}=0,325
$$

nous avons trouvé un $\beta_{z(\exp )}$ moyen de 0,27. Cette différence entre les deux valeurs de $\beta_{z}$ peut être due à la présence d'une charge d'espace ionique importante (cf. § 2.7), qui se manifeste par l'introduction d'un terme $\Delta a_{\mathrm{CE}}$. Nous avons alors la relation approchée :

$$
\beta_{\exp } \approx\left(a+\Delta a_{\mathrm{CE}}+q^{2} / 2\right)^{1 / 2}
$$

d'où :

$$
\Delta a_{\mathrm{CE}}=\beta_{\exp }^{2}-\beta_{\text {théor. }}^{2}=-0,0327 .
$$

D'après la relation (28), la densité ionique peut s'exprimer en fonction de $\Delta a_{\mathrm{CE}}$ :

$$
N_{\mathrm{i}}=-\frac{3}{8} \frac{M \Omega^{2} \varepsilon_{0}}{(Z e)^{2}} \Delta a_{\mathrm{CE}}
$$

soit, pour les ions d'argon monochargés :

$$
N_{\mathrm{i}}=-5,4 \times 10^{13} \Delta a_{\mathrm{CE}}=1,8 \times 10^{12} / \mathrm{m}^{3} .
$$

Cette valeur est du même ordre de grandeur que celles trouvées par Fischer [2] pour le krypton et Dehmelt-Major [9] pour $\mathrm{H}_{2}^{+}$, soit $4 \times 10^{12} / \mathrm{m}^{3}$ et $10^{11} / \mathrm{m}^{3}$ respectivement.

Appliquant la méthode de détection décrite ci-dessus, nous avons d'abord fait des mesures relatives du nombre d'ions confinés de charge unité en fonction de la durée d'ionisation $\tau_{\mathrm{i}}$ et de la durée de confinement $\tau_{\mathrm{c}}$. Ces temps sont définis de la façon suivante :

- au temps $t_{0}$ : mise en fonctionnement du faisceau électronique et de la tension de confinement ;

- au temps $t_{1}$ : coupure du faisceau, par blocage par le wehnelt ;

- au temps $t_{2}$ : application de la tension de détection à $f_{z}$. On a alors :

$$
\begin{aligned}
& \tau_{\mathrm{i} 0}=t_{1}-t_{0} \\
& \tau_{\mathrm{c}}=t_{2}-t_{1} .
\end{aligned}
$$

En réalité, la détection ne se fait pas de façon instantanée au temps $t_{2}$. Elle se fait en appliquant la tension à $f$ e et en même temps en démarrant le balayage en $V$ (ou en $U_{0}$ ). La vitesse de balayage doit être uniforme et identique pour toutes les mesures, car c'est elle qui détermine le temps pendant lequel est appliqué la tension de détection, et par conséquent le nombre d'ions détectés. La fréquence de balayage est de l'ordre de $0,1 \mathrm{~Hz}$.

La figure 13 représente la hauteur $h$ du signal détecté, c'est-à-dire une grandeur proportionnelle au nombre d'ions confinés, en fonction de la durée $\tau_{\mathrm{i} 0}$ d'ionisation des atomes d'argon par le faisceau, à $\tau_{c}$ constant. La partie linéaire croissante de la courbe correspond à l'accroissement du nombre d'ions piégés, suivant la loi de création :

$$
\frac{\mathrm{d} N}{\mathrm{~d} t}=\sigma_{0-1} N_{0} N_{\mathrm{e}} v
$$

soit

$$
N=\sigma_{0-1} N_{0} N_{\mathrm{e}} v t
$$

avec $N_{0}=$ densité de neutres; $N_{\mathrm{e}}=$ densité d'électrons rapides; $v=$ vitesse des électrons rapides; $\sigma_{0-1}=$ section efficace d'ionisation des neutres en ions une fois chargée.

La seconde partie de la courbe est un palier; elle indique que la charge ionique maximale pouvant être piégée est atteinte, pour les valeurs données de la pression et du courant dans le faisceau.

La figure 14 donne $h$ en fonction de la durée de confinement $\tau_{\mathrm{c}}$, avec $\tau_{\mathrm{i} 0}$ constant et égal à $30 \mathrm{~s}$, les autres paramètres étant inchangés par rapport au cas précédent. La courbe est décroissante et présente deux parties de caractères différents. En effet, la diminution dans le temps du nombre des particules piégées peut être due à deux termes de perte. Le premier, le plus 


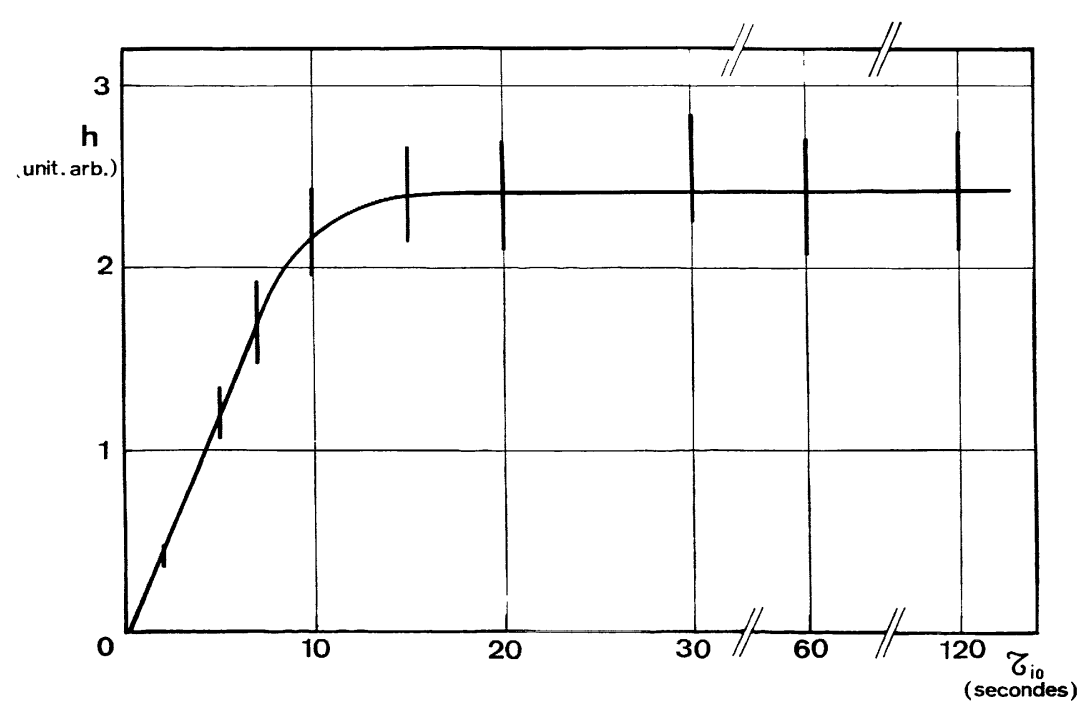

Fig. 13. - Variation de signal de détection en fonction de la durée d'ionisation par le faisceau, pour $Z=0 \rightarrow 1$.

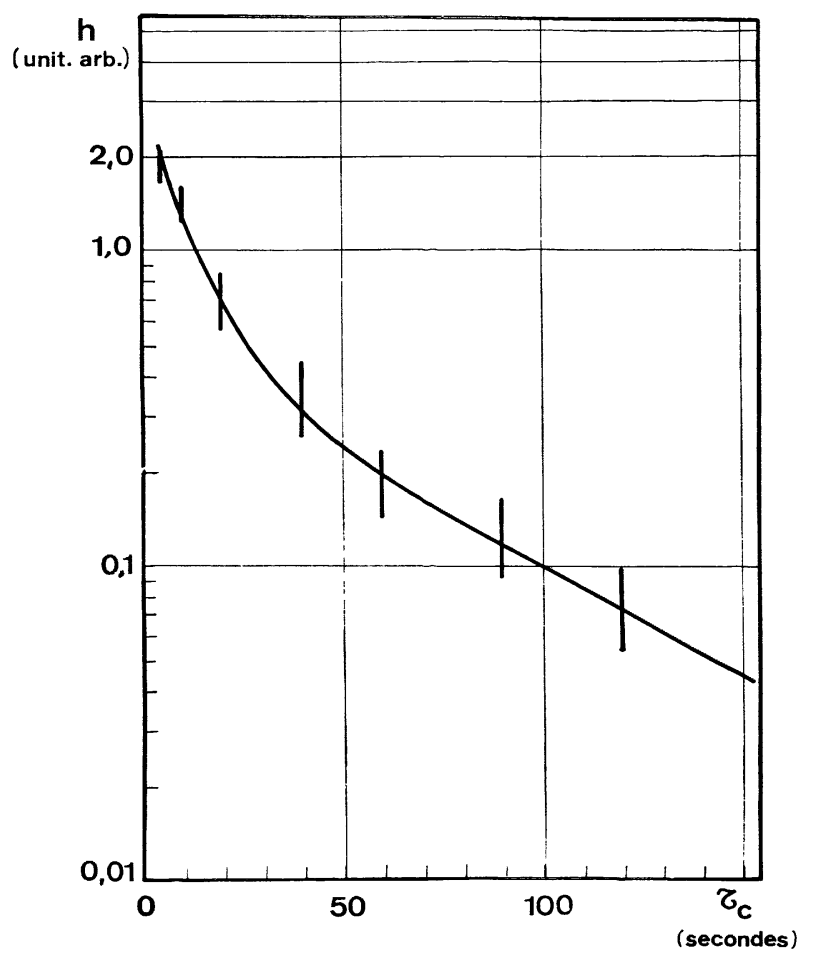

Fig. 14. - Variation du signal de détection en fonction de la durée de confinement, pour $Z=0 \rightarrow 1$.

important au départ, provient de la diffusion ion-ion, comme conséquence de la charge d'espace ionique. Il est de la forme :

$$
\frac{\mathrm{d} N}{\mathrm{~d} t}=-A N^{2}
$$

soit

$$
N \propto \alpha t^{-1} \quad(\alpha, A \text { sont des constantes }) .
$$

Cette loi de variation se vérifie bien pour $\tau_{\mathrm{c}} \leqslant 50 \mathrm{~s}$. Lorsque la densité ionique diminue, c'est le phéno- mène de la diffusion ion-neutre qui devient prépondérant. Il est donné par :

$$
\frac{\mathrm{d} N}{\mathrm{~d} t}=-\gamma N \quad \text { d'où } \quad N \propto \mathrm{e}^{-\gamma t}(\gamma=\mathrm{Cte}) .
$$

Sur le diagramme, tracé en coordonnées semilogarithmiques, nous trouvons bien une droite pour $\tau_{\mathrm{c}}>50 \mathrm{~s}$.

La durée maximale de piégeage que nous avons obtenue dans les mêmes conditions expérimentales que ci-dessus est de $5 \mathrm{~min}$. Nous pensons que la durée réelle doit être supérieure à cette valeur, car la méthode de détection que nous utilisons a une sensibilité limitée et contribue par ailleurs à la destruction partielle du piégeage.

La variation de $h$ avec la pression des neutres est représentée sur la figure 15. Aux faibles pressions, la densité ionique augmente avec la pression jusqu'à une limite imposée par l'équilibre entre le taux de création et le taux de perte due aux collisions ionsneutres. Ce dernier l'emporte aux fortes pressions et le nombre d'ions piégés diminue rapidement.

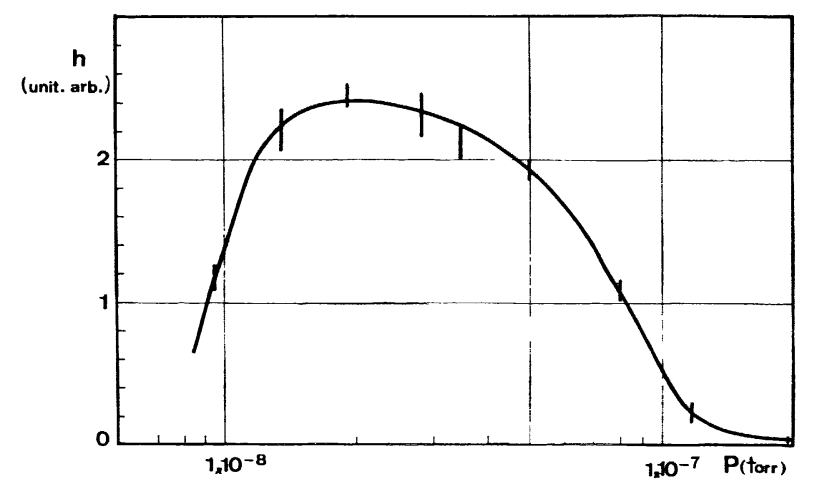

Fig. 15. - Variation du signal de détection en fonction de la pression pour $Z=0 \rightarrow 1$. 
La figure 16 montre un enregistrement d'un spectre de particules confinées, pour une fréquence de détection de $52 \mathrm{kHz}$ et en faisant un balayage en $V$. On peut y voir que les différents pics d'absorption sont obtenus pour des tensions dont les rapports correspondent sensiblement aux quatre premières charges de l'argon.

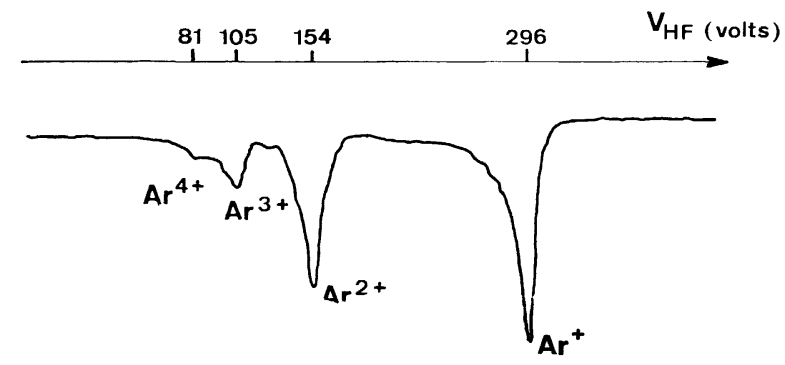

FIg. 16. - Enregistrement des quatre premières charges de l'argon.

4. Conclusion. - Le travail rapporté ici représente la première étape dans la réalisation d'un système permettant d'étudier la création et le confinement d'ions multichargés dans un champ quadrupolaire haute fréquence. L'étude théorique a mis en évidence le rôle important des vitesses initiales et des phases de création des ions, sur les conditions de confinement. Les mesures faites sur les ions monochargés sont en bon accord avec la théorie, et pour la première fois des ions multichargés ont pu être détectés avec un tel système de confinement.
Le procédé simple de détection utilisé ici souffre cependant de deux inconvénients majeurs. D’abord, en opérant à $f_{z}=f_{\mathrm{i}}=$ Cte, nous devons balayer en $V$ et en $U_{0}$, c'est-à-dire modifier les conditions de confinement des particules; ceci revient à attribuer à chaque charge et à chaque instant, de nouvelles conditions initiales qui risquent, comme nous l'avons vu dans la partie théorique (cf. $\$ 2.4$ ), de les rendre instables. Ensuite, pour augmenter la cohérence du mouvement ionique et le rendre plus aisément détectable, nous devons lui imposer un champ dipolaire externe de fréquence $f_{z}$, de valeur non négligeable. On démontre [10] que, dans ces conditions, l'amplitude des oscillations ioniques est fonction linéaire du temps, par conséquent la particule peut sortir rapidement du volume de confinement.

Cependant, la méthode est facile à mettre en œuvre et a permis, moyennant quelques précautions opératoires, de détecter les différents états de charge et d'étudier les caractéristiques du confinement. Dans un proche avenir, nous envisageons d'utiliser d'autres principes de détection, soit par exemple à fréquence de détection $f_{z}$ variable - ceci au prix d'une électronique plus sophistiquée - soit encore en éjectant l'ensemble des ions hors de la cage pour en faire ensuite une analyse par des moyens classiques. Ces modifications, ainsi que des améliorations apportées au dispositif expérimental, nous permettront de détecter des ions d'état de charge plus élevé et de mieux étudier le mécanisme de leur formation.

\section{Bibliographie}

[1] Paul, W., Reinhard, H. P. et von Zahn, U., Z. Phys. 152 (1958) 143.

[2] Fischer, E., Z. Phys. 156 (1959) 26.

[3] Rettinghaus, G., Z. Angew. Phys. 22 (1967) 321.

[4] Dawson, P. H. et Whetten, N. R., J. Vac. Sci. Technol. 5 (1968) 1.

[5] Baril, M. et Septier, A., Revue Phys. Appl. 9 (1974) 525.
[6] Bovet, G., Gouiran, R., Gumowski, I. et Reich, K. H., Rap. CERN/MPS-SI/Int (1970).

[7] Gaponov, A. V. et Miller, M. A., Sov. Phys. JetP 34 (1958) 515.

[8] Schwebel, C. E., Thèse de $3^{\mathrm{e}}$ cycle, Orsay (1974).

[9] Major, F. G. et Dehmelt, H. G., Phys. Rev. 170 (1968) 91.

[10] Kotowski, G., Z. Angew. Math. Mech. 23 (1943) 213. 